\title{
Resíduo de cevada industrial para a produção de painéis aglomerados
}

\author{
Industrial barley residue for the production of agglomerated panels \\ Helena Cristina Vieira*, Polliana D`Angelo Rios, Alexsandro Bayestorff da Cunha, Daniella Del \\ Castanhel Kniess, Angela Zakostelsky Neves Gaa, Gabriela Canarin Ricardo, Ailton Leonel Balduino \\ Junior
}

Universidade do Estado de Santa Catarina, Lages, SC, Brasil. *Autor para correspondência: lenacristin@hotmail.com

Submissão: 22/09/2018 / Aceite: 22/06/2020

\begin{abstract}
RESUMO
Neste trabalho objetivou-se avaliar as propriedades físicas e mecânicas de painéis aglomerados constituídos por resíduos de cevada industrial e partículas de Pinus spp. As partículas de Pinus spp. foram fornecidas pela empresa Bonet Madeiras e Papéis Ltda. localizada em Santa Cecília, Santa Catarina. O resíduo de cevada foi fornecido pela AMBEV, Lages, SC. Para a produção dos painéis foram utilizados cinco tratamentos, sendo compostos por diferentes teores de resíduo de cevada e de partículas de Pinus spp., respectivamente: 100:0 (T1), 75:25 (T2), 50:50 (T3), 25:75 (T4) e 0:100 (T5). Foram produzidos três painéis por tratamento, totalizando 15 painéis. Na produção dos painéis, utilizou-se a densidade nominal de $650 \mathrm{Kg} \cdot \mathrm{m}^{-3}, 12 \%$ de resina ureia formaldeído, $1 \%$ de parafina e ciclo de prensagem

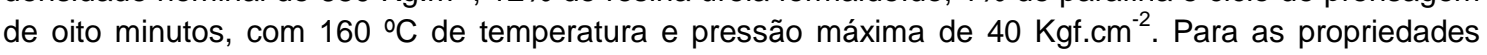
físicas determinou-se o teor de umidade, densidade, razão de compactação, inchamento em espessura e absorção de água ( $2 \mathrm{~h}$ e $24 \mathrm{~h}$ ). As propriedades mecânicas determinadas foram resistência a flexão estática, ligação interna, arrancamento de parafuso de topo e superfície. Com os resultados encontrados pode-se concluir que o aumento da porcentagem de resíduo de cevada diminui a resistência dos painéis para as propriedades físicas e mecânicas.
\end{abstract}

PALAVRAS-CHAVE: Hordeum vulgare, indústria cervejeira, MDP.

\begin{abstract}
This work aimed to evaluate the physical and mechanical properties based panels made of barley industrial waste and particles Pinus spp. The company Bonet Woods and Papers Ltda. in the municipality of Santa Cecilia - Santa Catarina, provided the particles Pinus spp. The barley residue was provided by AMBEV in Lages, SC. For the production of panels used were five treatments, being composed of different barley residue content and particle Pinus spp.: 100:0 (T1), 75:25 (T2), 50:50 (T3), 25:75 (T4) and 0:100 (T5), to the residue and timber respectively. Three panels were produced per treatment, totaling 15 panels. The nominal density of $650 \mathrm{Kg} \cdot \mathrm{m}^{-3}, 12 \%$ of urea formaldehyde resin, $1 \%$ paraffin, eight minutes press cycle were used in the production of panels, with a temperature of $160{ }^{\circ} \mathrm{C}$ and a maximum pressure of 40 Kgf. $\mathrm{cm}^{-2}$. For the physical properties, moisture content, density, compression ratio, thickness swelling and water absorption ( $2 \mathrm{~h}$ and $24 \mathrm{~h}$ ) were determined. The mechanical properties determined were static bending, internal connection, top and surface screw pulling. With the results found it can be concluded that increasing the percentage of barley residue decreases the resistance of the panels to the physical and mechanical properties.
\end{abstract}

KEYWORDS: Hordeum vulgare, brewing industry, MDP.

\section{INTRODUÇÃO}

Visando o melhor aproveitamento de resíduos, diversos trabalhos mostram que, além da madeira, outras fontes de fibras podem ser utilizadas na confecção de painéis aglomerados como: bambu (CALEGARI et al. 2007, MORAIS et al. 2018), casca de arroz (MELO et al. 2009), bagaço de cana-deaçúcar (MENDES et al. 2010, FIORELLI et al. 2011, SOARES et al. 2017), coco babaçu (MACHADO et al. 2017) e fibra de coco verde (FIORELLI et al. 2015). Contudo, ainda não foi verificado o comportamento de painéis aglomerados produzidos com resíduos de cevada.

A cevada (Hordeum vulgare L.) é um cereal de inverno que ocupa a quinta posição, em ordem de 
importância econômica, no mundo. Segundo a Associação Brasileira da Indústria da Cerveja, o Brasil é o terceiro maior produtor de cervejas do mundo, atrás somente dos Estados Unidos e da China. A expectativa é de pequeno aumento na área de cevada no Brasil, chegando a 123 mil hectares segundo levantamento do IBGE (junho 2018). Contudo, a previsão de clima favorável deverá resultar em rendimento médio acima de $3 \mathrm{mil} \mathrm{kg/ha,} \mathrm{com} \mathrm{um} \mathrm{volume} \mathrm{de} \mathrm{produção} \mathrm{estimado} \mathrm{em} 427$ mil toneladas. A área de cevada no Brasil, chegando a 123 mil hectares segundo levantamento do IBGE (junho 2018). Contudo, a previsão de clima favorável deverá resultar em rendimento médio acima de $3 \mathrm{mil} \mathrm{kg} / \mathrm{ha}$, com um volume de produção estimado em 427 mil toneladas.

Em 2013, foram utilizadas mais de 378 mil toneladas de cevada no Brasil, para produzir aproximadamente 13,4 bilhões de litros de cerveja. A produção cresce cerca de $25,8 \%$ ao ano e atualmente, a disponibilidade do bagaço de cevada no Brasil ultrapassa 2.000 mil toneladas/ano (CERVBRASIL 2016).

A produção brasileira se concentra na Região Sul, que contribuiu em 2019 com 98\% da produção nacional do cereal, seguido pela Região Sudeste, com registro de $2 \%$ de cultivo (IBGE 2020).

Para as indústrias de cerveja, o bagaço de cevada é considerado um problema, uma vez que é necessário descartar o resíduo sem agredir o ambiente, para não causar impacto negativo (BROCHIER 2007). De acordo com BROCHIER \& CARVALHO (2009), o resíduo úmido de cervejaria é resultante da fase inicial do processo de fabricação de cervejas e pode ser encontrado na forma de cascas ou de farelo. FREITAS (2006), afirma que para cada $100 \mathrm{Kg}$ de malte utilizado na fabricação de cerveja são gerados entre 118 e $130 \mathrm{Kg}$ de bagaço de cevada com $79 \%$ de umidade, sendo esse o principal fator limitante à sua utilização (LIMA 1993).

Assim, visando aumentar o aproveitamento do resíduo gerado na indústria cervejeira, este trabalho teve como objetivo avaliar as propriedades físicas e mecânicas de painéis aglomerados constituídos por resíduos de cevada industrial em associação com partículas de Pinus spp. em diferentes proporções.

\section{MATERIAL E MÉTODOS}

\section{Coleta e preparação do material}

Para a confecção dos painéis utilizou-se partículas de Pinus spp. fornecidas pela empresa Bonet Madeiras e Papéis Ltda. localizada no Município de Santa Cecília, Santa Catarina. O bagaço da cevada foi coletado na Companhia de Bebidas das Américas, Ambev S.A, em Lages, Santa Catarina.

\section{Delineamento experimental}

O delineamento utilizado foi de cinco tratamentos, com três repetições por tratamento, totalizando a produção de 15 painéis; utilizando a densidade nominal de $650 \mathrm{Kg} \cdot \mathrm{m}^{-3}$ e dimensões de $400 \times 400 \times 16 \mathrm{~mm}$. Foram confeccionados painéis homogêneos, seguindo a descrição da Tabela 1.

Tabela 1. Composição dos painéis.

Table 1. Composition of panels.

\begin{tabular}{ll}
\hline Tratamento & Composição \\
\hline T1 - C 100 P 0 & 100\% Bagaço de Cevada \\
T2 - C 75 P 25 & 75\% Bagaço de Cevada e 25\% Partículas de Pinus spp. \\
T3 - C 50 P 50 & 50\% Bagaço de Cevada e 50\% Partículas de Pinus spp. \\
T4 - C 25 P 75 & 25\% Bagaço de Cevada e 75\% Partículas de Pinus spp. \\
T5 - C 0 P 100 & 100\% Partículas de Pinus spp. \\
\hline
\end{tabular}

Onde: $\mathrm{C}$ = Bagaço de Cevada, $\mathrm{P}$ = Partículas de Pinus spp.

Cabe salientar, que o tratamento composto por painéis produzidos com $100 \%$ partículas de Pinus spp. foi utilizado como referência. Uma vez que essa é a composição usual da indústria de painéis de partículas.

\section{Preparação dos painéis}

Para a confecção dos painéis foram utilizados resíduos de cevada com densidade média de 220 $\mathrm{Kg} \cdot \mathrm{m}^{-3}$ e partículas de Pinus spp. de $410 \mathrm{Kg} \cdot \mathrm{m}^{-3}$. Todas as partículas foram secas em estufa de circulação forçada de ar a temperatura de $60 \pm 2{ }^{\circ} \mathrm{C}$. Para a produção dos painéis utilizou-se somente partículas com umidade inferior a $5 \%$. Os painéis foram produzidos no Laboratório de Tecnologia da Madeira do Departamento de Engenharia Florestal, na Universidade do Estado de Santa Catarina.

Utilizou-se $12 \%$ de adesivo ureia formaldeído e $1 \%$ de parafina, com teor de sólidos de, respectivamente, 70 e de $65 \%$. A aspersão do adesivo e da parafina nas partículas ocorreu em uma 
encoladeira tipo tambor giratório, dotado de um copo graduado e uma pistola a ar comprimido.

Para a prensagem foram realizadas duas fases distintas. Sendo a primeira fase a pré-prensagem a frio, utilizando uma prensa hidráulica para a acomodação das partículas na caixa formadora. A segunda fase foi a prensagem a quente, o ciclo utilizado foi efetuado em uma prensa hidráulica automática, modelo MA 098. A temperatura de prensagem foi de $160 \stackrel{\circ}{\circ}$, com tempo de oito minutos e pressão de $40 \mathrm{Kgf.cm}^{-2}$.

Após a confecção, os painéis foram armazenados em sala de climatização, seguindo a norma da NBR 14660 (ABNT 2004).

\section{Testes físicos e mecânicos}

Para os testes físicos e mecânicos foram utilizados corpos de prova com dimensões padronizadas pelas normas, conforme está representado na Tabela 2.

Tabela 2. Dimensões dos corpos de prova utilizados para os testes físicos e mecânicos.

Table 2. Dimensions of the specimens used for the physical and mechanical tests.

\begin{tabular}{lcc}
\hline \multicolumn{1}{c}{ Propriedade avaliada } & Dimensões dos corpos de prova & Normas utilizadas \\
\hline Densidade & $50 \times 50 \mathrm{~mm}$ & ASTM D1037 (1999) \\
\hline Absorção de água 2 e 24 horas & $125 \times 125 \mathrm{~mm}$ & ASTM D1037 (1999) \\
\hline Inchamento em espessura 2 e 24 horas & & ASTM D1037 (1999) \\
\hline Ligação interna & $50 \times 50 \mathrm{~mm}$ & \multirow{2}{*}{ DIN 52362 (1982) } \\
\hline Módulo de elasticidade à flexão estática (MOE) & $250 \times 50 \mathrm{~mm}$ & NBR 14810 (ABNT \\
\hline Módulo de ruptura à flexão estática (MOR) & $250 \times 50 \mathrm{~mm}$ & 2013) \\
\hline Arrancamento de parafuso de topo & $125 \times 50 \mathrm{~mm}$ & \\
\hline Arrancamento de parafuso de superfície & &
\end{tabular}

A razão de compactação foi determinada utilizando a equação a seguir:

$R c=\frac{D p}{D m}$

Onde: $\mathrm{Rc}=$ Razão de compactação;

$\mathrm{Dp}=$ Densidade dos painéis;

$\mathrm{Dm}=$ Densidade do material lignocelulósico.

O teor de umidade foi determinado em corpos de prova com dimensões de $50 \times 50 \times 16 \mathrm{~mm}$ (largura, comprimento e espessura). Para perda total de umidade, os corpos de prova foram levados para estufa de circulação forçada a $100 \stackrel{\circ}{\mathrm{C}}$ até massa constante.

Os resultados médios das diferentes composições foram comparados com as normas de qualidade: NBR 14810 (ABNT 2013), ANSI A208.1 (1993), CS 236-66 (COMMERCIAL STANDART 1968) e EN 312-2 (EUROPPEAN STANDART 2003).

\section{Análise estatística}

Os tratamentos utilizados foram considerados qualitativos, portanto, para todas as variáveis foi utilizado o Delineamento Inteiramente Casualizado (DIC). Após a confirmação da normalidade e homogeneidade dos dados, as análises estatísticas foram feitas no Sisvar ${ }^{\circledR}$ - Sistema de análise de variância (FERREIRA 2000), ao nível de 5\% de probabilidade para o teste de Scott-Knott.

\section{RESULTADOS E DISCUSSÃO \\ Propriedades Físicas}

Os valores médios para densidade, razão de compactação, espessura e teor de umidade dos painéis estão apresentados na Tabela 3, juntamente com os seus coeficientes de variação.

Os valores médios para densidade dos painéis variaram de 620 à $680 \mathrm{Kg} \cdot \mathrm{m}^{-3}$. Pode-se observar ainda que apenas os painéis com $100 \%$ cevada diferenciaram-se estatisticamente das demais composições.

Com relação aos requisitos mínimos exigidos pelas normas de qualidade para classificação dos painéis, verifica-se que os mesmos podem ser definidos como de média densidade, considerando a NBR 14810 (ABNT 2013) e a CS 236-66 (COMMERCIAL STANDART 1968). Contudo, segundo as especificações da ANSI A208.1 (1993), apenas os painéis compostos 100\% cevada e $100 \%$ partículas de Pinus spp. podem ser classificados como de média densidade, pois encontram-se acima do valor mínimo de 
$640 \mathrm{Kg} \cdot \mathrm{m}^{-3}$, já os painéis abaixo desse valor, T2 T3 e T4, podem ser considerados de baixa densidade.

Tabela 3. Valores médios de densidade, razão de compactação, teor de umidade e espessura.

Table 3. Mean density values, compression ratio, moisture content and thickness.

\begin{tabular}{llll}
\hline Composição & D.E. $\left(\right.$ Kg.m $\left.{ }^{-3}\right)$ & Razão de Compactação & \multirow{2}{*}{ Umidade $(\%)$} \\
\hline C 100 P 0 & $680_{(6,13)} \mathrm{a}$ & $3,10_{(6,13)} \mathrm{e}$ & $10,25_{(2,88)} \mathrm{C}$ \\
C 75 P 25 & $620_{(3,44)} \mathrm{b}$ & $2,33_{(3,44)} \mathrm{d}$ & $9,78_{(1,52)} \mathrm{b}$ \\
C 50 P 50 & $620_{(4,39)} \mathrm{b}$ & $1,97_{(4,39)} \mathrm{C}$ & $9,34_{(7,72)} \mathrm{a}$ \\
C 25 P 75 & $630_{(5,20)} \mathrm{b}$ & $1,79_{(5,2))} \mathrm{b}$ & $9,16_{(3,08)} \mathrm{a}$ \\
C 0 P 100 & $650_{(3,91)} \mathrm{b}$ & $1,55_{(3,91)} \mathrm{a}$ & $9,26_{(2,15)} \mathrm{a}$ \\
\hline Média & 640 & 2,15 & 9,56 \\
\hline
\end{tabular}

Legenda: $\mathrm{C}$ = Cevada, $\mathrm{P}=$ Pinus spp., D.E. = Densidade, subscrito = coeficiente de variação (\%). Médias seguidas de mesma letra na coluna não diferem estatisticamente entre si.

Com relação a razão de compactação, apenas os painéis produzidos com $100 \%$ de partículas de Pinus spp. apresentaram valores médios no intervalo recomendado por KELLY (1977), MOSLEMI (1974) e MALONEY (1993) na faixa de 1,3 a 1,6. A alta razão de compactação observada nos tratamentos com bagaço de cevada se deve a baixa densidade do material $\left(220 \mathrm{Kg} \cdot \mathrm{m}^{-3}\right)$, em comparação com a madeira de Pinus spp. (410 Kg.m³). Assim, foi possível perceber que a razão de compactação aumenta conforme é adicionada uma maior porcentagem de cevada na associação com as partículas de Pinus spp.

O valor médio de teor de umidade variou de $9,26 \%$ a $10,25 \%$. Salienta-se que os valores encontrados estão abaixo da umidade de equilíbrio da sala de climatização que é de $12 \%$ para madeiras. A diminuição da higroscopicidade dos painéis ocorre em função da incorporação de resina e parafina (WU 1999), além das altas temperaturas aplicadas durante a etapa de prensagem, que fazem com que a pressão compacte as camadas externas do painel, tornando os sítios de adsorção de água menos disponíveis (DEL MENEZZI 2004).

Ainda, sobre o teor de umidade dos painéis, nota-se que os valores médios de todas as composições estão de acordo com o especificado pela NBR 14810 (ABNT 2013), que define intervalo entre de 5\% a $11 \%$. Para a ANSI A208.1 (1993), que pressupõe uma umidade de 10\%, apenas os painéis compostos $100 \%$ com partículas de cevada não atendem a norma.

$\mathrm{Na}$ Tabela 4, estão apresentados os valores médios de absorção de água e inchamento em espessura após duas e 24 horas de imersão em água. Para absorção de água, denota-se que os painéis compostos $100 \%$ de partículas de madeira foram os que apresentaram o menor valor médio, tanto após o primeiro ciclo de imersão (12,45\%), quanto após o segundo (32,05\%). Todos os painéis produzidos em associação com a cevada apresentaram valores estatisticamente maiores que o composto por $100 \%$ Pinus spp. A maior absorção para 2 horas foi observada nos painéis com $100 \%$ cevada. Para 24 horas os painéis produzidos com $100 \%$ de partículas de Pinus spp. foram estatisticamente superiores em qualidade que as demais composições, uma vez que apresentaram menor absorção de água.

Tabela 4. Valores médios para absorção de água e inchamento em espessura.

Table 4. Mean values for water absorption and thickness swelling.

\begin{tabular}{lllll}
\hline \multirow{2}{*}{ Composição } & \multicolumn{3}{c}{ A.A. $(\%)$} & \multicolumn{2}{c}{ I.E. $(\%)$} \\
\cline { 2 - 5 } & 2 horas & 24 horas & 2 horas & 24 horas \\
\hline C 100 P 0 & $72,40_{(5,48)} \mathrm{d}$ & $89,53_{(13,45)} \mathrm{b}$ & $16,67_{(5,81)} \mathrm{b}$ & $22,24_{(8,15)} \mathrm{b}$ \\
C 75 P 25 & $56,44_{(3,77)} \mathrm{C}$ & $79,97_{(6,51)} \mathrm{b}$ & $13_{1,15_{(3,94)} \mathrm{b}}$ & $21,09_{(7,76)} \mathrm{b}$ \\
C 50 P 50 & $52,45_{(3,13)} \mathrm{C}$ & $75,23_{(7,97)} \mathrm{b}$ & $14,63_{(4,13)} \mathrm{b}$ & $20,27_{(7,40)} \mathrm{b}$ \\
C 25 P 75 & $32,27_{(2,59)} \mathrm{b}$ & $67,69_{(3,08)} \mathrm{b}$ & $9,03_{(4,89)} \mathrm{a}$ & $18,32_{(7,07)} \mathrm{b}$ \\
C 0 P 100 & $12,45_{(3,46)} \mathrm{a}$ & $32,05_{(4,13)} \mathrm{a}$ & $7,74_{(1,28)} \mathrm{a}$ & $13,35_{(4,94)} \mathrm{a}$ \\
\hline Média & 12,24 & 45,20 & 19,06 & 68,89 \\
\hline
\end{tabular}

Legenda: $\mathrm{C}=$ Cevada, $\mathrm{P}=$ Pinus spp., A.A $=$ absorção de água, I.E.= inchamento em espessura, subscrito = coeficiente de variação (\%). Médias seguidas de mesma letra na coluna não diferem estatisticamente entre si.

Pode-se observar que o aumento na proporção de resíduo de cevada é diretamente proporcional ao aumento no valor de absorção de água. Esta tendência também foi observada por MELO et al. (2009) para 
painéis produzidos com diferentes proporções de madeira e casca de arroz e MENDES et al. (2012) na produção de painéis de madeira em associação com bagaço de cana.

Os resultados obtidos para absorção de água são maiores aos encontrados em outras pesquisas. TRIANOSKI (2010) definiu valores de 7,94\% para duas horas e $27,82 \%$ para 24 horas em painéis de partículas homogêneos de Pinus taeda com 12\% de resina. MENDES et al. (2010), em seu estudo com painéis produzidos com $75 \%$ de bagaço de cana e $12 \%$ de resina, encontraram valores de $21,56 \%$ para absorção de água após 24 horas de imersão.

Porém, outras pesquisas determinaram valores superiores de absorção de água, como BATISTA et al. (2007) que avaliaram as propriedades físicas de painéis aglomerados de três camadas de Pinus elliottii Engelm. produzidos com 1\% de parafina e $6 \%$ de ureia-formaldeído e encontraram valores de absorção de água de 150,6 e 168,9\% após duas e 24 horas, respectivamente. Ainda MENDES et al. (2009) obtiveram valores de absorção de água após 24 horas de 95,78\% e 104,56\% para painéis aglomerados de 700 Kg.m ${ }^{3}$ com $1 \%$ de parafina produzidos com Eucalyptus urophylla de sete e 12 anos de idade, respectivamente.

Para a variável inchamento em espessura após duas horas, os painéis compostos $100 \%, 75 \%$ e $50 \%$ cevada foram superiores aos demais. Considerando o inchamento em 24 horas de imersão em água, apenas o painel produzido com 100\% partícula de pinus apresentou-se superior, ou seja, inchou menos em espessura.

Em relação ao atendimento aos critérios das normas de qualidade, verifica-se que segundo a NBR 14810 (ABNT 2013), somente os painéis produzidos com 100\% partículas de Pinus spp. atendem ao valor máximo de $8 \%$ para inchamento em espessura após duas horas em imersão de água.

Quanto ao inchamento 24 horas, todas as composições se enquadram nas normas CS 236-66 (COMMERCIAL STANDART 1968) e na ANSI A208.1 (1993) que estabelecem valores máximos de, respectivamente, 35\% e 40\%. Já em relação a NBR 14810 (ABNT 2013) e a EN 312-2 (EUROPPEAN STANDART 2003), apenas os painéis produzidos com 100\% partículas de Pinus spp. atenderam as normas, as quais estabelecem valores máximos de 18 e $15 \%$ respectivamente.

De acordo com os dados obtidos, verificou-se que o tratamento de maior destaque para ambas as faixas de inchamento em espessura foi o de $100 \%$ partículas de Pinus spp. Porém o pré-tratamento das partículas de cevada pode ser estudado em trabalhos futuros, com o objetivo de diminuir as porcentagens de absorção e inchamento em duas e 24 horas.

\section{Propriedades Mecânicas}

Os valores médios para as propriedades de flexão estática estão apresentados na Tabela 5.

Tabela 5. Valores médios para flexão estática.

Table 5. Medium values for static bending.

\begin{tabular}{lll}
\hline \multirow{2}{*}{ Composição } & Flexão Estática (MPa) & \\
\cline { 2 - 3 } & MOE & MOR \\
\hline C 100 P 0 & $267,93_{(21,22)} \mathrm{e}$ & $2,15_{(32,72)} \mathrm{e}$ \\
C 75 P 25 & $627,81_{(13,40)} \mathrm{d}$ & $6,27_{(13,81)} \mathrm{d}$ \\
C 50 P 50 & $751,96_{(18,17)} \mathrm{C}$ & $7,92_{(23,06)} \mathrm{C}$ \\
C 25 P 75 & $1007,31_{(15,53)} \mathrm{b}$ & $11,20_{(19,93)} \mathrm{b}$ \\
C 0 P 100 & $1368,48_{(11,30) \mathrm{a}}$ & $15,58_{(15,95) \mathrm{a}}$ \\
\hline Média & 814,86 & 8,62 \\
\hline
\end{tabular}

Legenda: $\mathrm{C}=$ Cevada, $\mathrm{P}=$ Pinus spp., $\mathrm{MOE}=$ Módulo de elasticidade, $\mathrm{MOR}=$ módulo de ruptura, sobrescrito $=$ tratamento, subscrito = coeficiente de variação (\%). Médias seguidas de mesma letra na coluna não diferem estatisticamente entre si.

Para os valores de MOE, observa-se que as médias variaram entre 267,93 MPa para a composição $100 \%$ cevada, e $1368,48 \mathrm{MPa}$ para a composição $100 \%$ partículas de Pinus spp. Para o MOR os valores variaram de 2,15 a $15,58 \mathrm{MPa}$ respetivamente, para as composições $100 \%$ cevada e $100 \%$ Pinus spp.

Para ambas as propriedades, todos os tratamentos foram estatisticamente diferentes. $O$ acréscimo de partículas de cevada ocasionou uma redução das propriedades de MOE e MOR. Este comportamento, resultante do acréscimo de partículas do material alternativo, também foi observado para painéis produzidos com casca de arroz (MELO et al. 2009), diferentes porcentagens de bagaço de cana-de-açúcar (MENDES et al. 2010) e ao adicionar feixes de sisal na produção dos painéis aglomerados (MESQUITA et al. 2015).

Em comparação aos critérios exigidos pelas normas de qualidade, a NBR 14810 (ABNT 2013) 
estabelece valores de MOE e de MOR mínimos de $1600 \mathrm{MPa}$ e $11 \mathrm{MPa}$. Neste estudo, para a propriedade de MOE, nenhuma composição atendeu os requisitos estabelecidos. Já para MOR, apenas as composições $75 \%$ e 100\% partículas de Pinus spp. atenderam o mínimo requerido.

Para a EN 312-2 (EUROPPEAN STANDART 2003), a qual estabelece valores mínimos de referência para o MOE de $1600 \mathrm{MPa}$, nenhuma composição atendeu o requerido. Enquanto o MOR, no qual são exigidos valores mínimos de $13 \mathrm{MPa}$, apenas a composição $100 \%$ partículas de Pinus spp. atingiu o solicitado pela norma.

No ensaio de ligação interna, os valores médios variaram de 0,10 a 0,43 Mpa, conforme a Tabela 6.

Tabela 6. Valores médios referentes à ligação interna.

Table 6. Mean values for the internal connection.

\begin{tabular}{ll}
\hline Composição & Ligação interna (MPa) \\
\hline C 100 P 0 & $0,10_{(32,79)} \mathrm{d}$ \\
C 75 P 25 & $0,28_{(29,79)} \mathrm{c}$ \\
C 50 P 50 & $0,42_{(32,17)} \mathrm{b}$ \\
C 25 P 75 & $0,43_{(30,22)} \mathrm{b}$ \\
C 0 P 100 & $0,55_{(20,18)} \mathrm{a}$ \\
\hline Média & 0,36
\end{tabular}

Legenda: $\mathrm{C}=$ Cevada, $\mathrm{P}=$ Pinus spp., sobrescrito $=$ tratamento, subscrito $=$ coeficiente de variação (\%). Médias seguidas de mesma letra na coluna não diferem estatisticamente entre si.

O comportamento de queda da resistência na ligação interna resultante do acréscimo de resíduo industrial na produção de painéis aglomerados, também foi observado por MENDES (2010) e MELO et al. (2009) em seus estudos com partículas de bagaço de cana de açúcar e de casca de arroz, respectivamente.

Com relação as normas de qualidade, a NBR 14810 (ABNT 2013) e a EN 312 (EUROPPEAN STANDART 2003), as quais estabelecem valores mínimos de $0,35 \mathrm{MPa}$, neste estudo, apenas as composições de $50 \%$, $75 \%$ e $100 \%$ partículas de Pinus spp. atingiram as normas. Para a CS $236-66$ (COMMERCIAL STANDART 1968) a qual determina 0,48 MPa, apenas a composição $100 \%$ partículas de Pinus spp. atendeu o mínimo exigido.

Já em relação a norma ANSI A208.1 (1993), que estabelece classes de qualidade: M-1 e MS (0,36 MPa), M-2 (0,40 MPa) e M-3i (0,50 Mpa), as composições 50\% e $25 \%$ cevada são classificados como M-2, já a composição 100\% partículas de Pinus spp. ser classificado como M-3i. Os tratamentos C100 e C75, não são classificados pela norma.

Para o arrancamento de parafusos de topo e de superfície, todas as composições diferiram estatisticamente. Os valores para arrancamento de parafusos da superfície variaram de $381,36 \mathrm{~N}$ à 1635,75 $\mathrm{N}$ e os de topo de $183,00 \mathrm{~N}$ à 1405,92 N (Tabela 7).

Tabela 7. Valores médios para resistência ao arrancamento de parafuso.

Table 7. Mean values for the screw pullout resistance.

\begin{tabular}{lll}
\hline \multirow{2}{*}{ Composição } & \multicolumn{2}{l}{ Arrancamento de Parafuso $(\mathrm{N})$} \\
\cline { 2 - 3 } & Superfície & Topo \\
\hline C 100 P 0 & $381,36_{(30,54)} \mathrm{e}$ & $183,00_{(22,28)} \mathrm{e}$ \\
C 75 P 25 & $763,17_{(14,48)} \mathrm{d}$ & $442,00_{(27,07)} \mathrm{d}$ \\
C 50 P 50 & $929,17_{(14,27)} \mathrm{C}$ & $707,00_{(17,03) \mathrm{C}}$ \\
C 25 P 75 & $1227,17_{(19,43)} \mathrm{b}$ & $990,58_{(17,74)} \mathrm{b}$ \\
C 0 P 100 & $1635,75_{(13,91)} \mathrm{a}$ & $1405,92_{(15,45)} \mathrm{a}$ \\
\hline Média & 987,32 & 745,70 \\
\hline
\end{tabular}

Legenda: $\mathrm{C}=$ Cevada, $\mathrm{P}=$ Pinus spp., sobrescrito $=$ tratamento, subscrito $=$ coeficiente de variação (\%). Médias seguidas de mesma letra na coluna não diferem estatisticamente entre si.

Assim como para as demais propriedades estudadas, o acréscimo de bagaço de cevada diminuiu a resistência ao arrancamento de parafuso. A mesma tendência também foi observada por MELO et al. (2009) ao acrescentar casca de arroz nos painéis produzidos em seu estudo.

No que se refere às normas de qualidade, de acordo com a norma NBR 14810 (ABNT 2013), para 
arrancamento de parafuso de superfície e de topo, onde o mínimo requerido é 1020 e $800 \mathrm{~N}$, respectivamente, apenas os tratamentos compostos por $75 \%$ e $100 \%$ partículas de Pinus spp. atingiram o determinado.

No entanto, para os parâmetros mínimos requeridos pela ANSI A208.1 (1993) onde a carga mínima exigida é de $700 \mathrm{~N}$ para topo e $800 \mathrm{~N}$ para superfície na classe MS, $800 \mathrm{~N}$ e $900 \mathrm{~N}$ na M-2 e $900 \mathrm{~N}$ e 1000 $\mathrm{N}$ na M-3i. A composição $50 \%$ bagaço de cevada pode ser classificada como MS, as composições $75 \%$ e 100\% partículas de Pinus spp. são consideradas da classe M-3i, já as composições 100\% e 75\% bagaço de cevada não podem ser classificadas em nenhuma classe.

\section{CONCLUSÃO}

Com os resultados encontrados pode-se concluir que o aumento da porcentagem de resíduo de cevada elevou a absorção de água e o inchamento em espessura. Além disso, é possível afirmar que o aumento da proporção de bagaço de cevada diminuiu a resistência mecânica dos painéis aglomerados produzidos.

Maiores proporções de adesivo e parafina, além do pré-tratamento do resíduo de cevada podem melhorar as propriedades físicas dos painéis. Por isso, novas pesquisas para determinar a viabilidade da produção de painéis utilizando resíduo de cevada da indústria cervejeira devem ser realizadas.

\section{REFERÊNCIAS}

ANSI. 1993. American National Standards Institute. A 208.1: Mat formed wood particleboard: specifications. Gaithersburg: National Particleboards Association. 9p.

ABNT. 2013. Associação Brasileira de Normas Técnicas. NBR 14810-2: Painéis de partícula de média densidade. Parte 2: requisitos e métodos de ensaio. $69 p$.

ABNT. 2004. Associação Brasileira de Normas Técnicas. NBR 14660: Madeira - Amostragem e preparação para análise. $7 p$.

ASTM 1999. American Society of Testing and Materials. Standard Test Method for Evaluating Properties of Wood-Base Fiber and Particle Panel Materials. Designation: ASTM D1037-99. 31p.

BATISTA DC et al. 2007. Fabricação de aglomerados de três camadas com madeira de Pinus elliottii Emgelm. e casca de Eucalyptus pellita F. Muell. Cerne 13: 178-187.

BROCHIER MA \& CARVALHO S. 2009. Aspectos ambientais, produtivos e econômicos do aproveitamento de resíduo úmido de cervejaria na alimentação de cordeiros em sistema de confinamento. Ciência e Agrotecnologia 33: 13921399.

BROCHIER MA. 2007. Aproveitamento de resíduo úmido de cervejaria na alimentação de cordeiros confinados em fase de terminação. Dissertação (Mestrado em Qualidade Ambiental). Novo Hamburgo: FEEVALE. 120p.

CALEGARI L et al. 2007. Desempenho físico-mecânico de painéis fabricados com bambu (Bambusa vulgaris Schr.) em combinação com madeira. Cerne 13: 57-63.

CERVBRASIL. 2014. Indústria cervejeira está conectada com o desenvolvimento do país. Disponível em: http://cervbrasil.org.br/2014/04/a-cerveja-como-contribuicao-economica/. Acesso em: 03 ago. 2020.

COMMERCIAL STANDART. 1968. CS 236-66: Mat formed wood particleboard. 18p.

DEL MENEZZI CHS. 2004. Estabilização dimensional por meio do tratamento térmico e seus efeitos sobre as propriedades de painéis de partículas orientadas (OSB). Tese (Doutorado em Engenharia Florestal). Curitiba: UFPR. 226p.

DIN. 1982. Normen, für holzfaserplaten spanplatten sperrholz. DIN 52362-Testing of wood chipboards, bending test, determination of bending strength. 40p.

EUROPPEAN STANDART. 2003. EN 312: Particleboards. Specifications. 17p.

FERREIRA DF. 2000. SisVar ${ }^{\circledR}$ : Sistema de análise de variância para dados balanceados, versão 4.0. Lavras: DEX/UFLA. (Software estatístico).

FIORELLI J et al. 2015. Painéis de partículas monocamadas fabricados com resíduo de madeira e fibra de coco verde. Scientia Forestalis 43: 175-182.

FIORELLI $\mathrm{J}$ et al. 2011. Painéis de partículas à base de bagaço de cana e resina de mamona - produção e propriedades. Acta Scientiarum. Technology 33: 401-406.

FREITAS GLS. 2006. Potencial antioxidante e compostos fenólicos na cerveja, chopp, cevada e no bagaço de brassagem. Dissertação (Mestrado em Ciências dos Alimentos). Florianópolis: UFSC. 86p.

IBGE. 2020. Instituto Brasileiro de Geografia e Estatística - Janeiro 2020. Levantamento sistemático da produção agrícola. 89p.

KELLY MW. 1977. Critical literature review of relationships between processing parameters and physical properties of particleboard. General Technical Report 10: 1-66.

LIMA MLM. 1993. Resíduo de cervejaria úmido: Formas de conservação e efeitos sobre parâmetros ruminais. Dissertação (Mestrado em Ciência Animal e Pastagem) Piracicaba: ESALQ/USP. 98p. 
MACHADO NAF et al. 2017. Painéis aglomerados fabricados com resíduos do coco babaçu. Revista Brasileira de Ciências Agrárias 12: 202-209.

MALONEY TM. 1993. Modern particleboard and dry-process fiberboard manufacturing. San Francisco: M. Freeman.

MELO RR et al. 2009. Propriedades físico-mecânicas de painéis aglomerados produzidos com diferentes proporções de madeira e casca de arroz. Ciência Florestal 19: 449-460.

MENDES LM et al. 2012. Efeito da associação de bagaço de cana, do tipo e do teor de adesivo na produção de painéis aglomerados. Ciência Florestal 22: 161-170.

MENDES LM et al. 2009. Eucalyptus urophylla stands wood utilization at two different ages for production of particleboard panels. Cerne 15: 288-294.

MENDES RF. 2010. Efeito do tratamento térmico sobre as propriedades de painéis OSB. Dissertação (Mestrado em Ciências). Piracicaba: ESALQ/USP. 115p.

MENDES RF et al. 2010. Painéis aglomerados produzidos com bagaço de cana em associação com madeira de eucalipto. Scientia Forestalis 38: 285-295.

MESQUITA RGDA et al. 2015. Inclusão de feixes de sisal na produção de painéis MDP de eucalipto. Scientia Forestalis 43: 75-82.

MORAIS WWC et al. 2018. Uso de Bambusa tuldoides e Eucalyptus grandis para confecção de painéis aglomerados. Ciência Florestal 28: 746-757.

MOSLEMI AA. 1974. Particleboard. Illinois: Southern Illinois University Press. 244p.

SOARES SS et al. 2017. Valorização do bagaço de cana-de-açúcar na produção de painéis aglomerados de baixa densidade. Revista Ciência da Madeira 8: 64-73.

TRIANOSKI R. 2010. Avaliação do potencial de espécies florestais alternativas, de rápido crescimento, para produção de painéis de madeira aglomerada. Dissertação (Mestrado em Ciências Florestais). Curitiba: UFPR. 262p.

WU Q. 1999. Application of Nelson's sorption isotherm to wood composites and overlays. Wood and Fiber Science 28: 227-239. 